\title{
A Mixture of the Exclusion Process and the Voter Model
}

\author{
Vladimir Belitsky ${ }^{1} \quad$ Pablo A. Ferrari ${ }^{1}$ \\ Mikhail V. Menshikov" ${ }^{* 1,2}$ Serguei Yu. Popov ${ }^{1,3}$
}

1 and the mailing address: Instituto de Matemática e Estatística, Universidade de São Paulo, Caixa Postal 66281, 05315-970 - São Paulo, SP - BRAZIL

${ }^{2}$ Faculty of Mathematics and Mechanics, Moscow State University

${ }^{3}$ Institute for Problems of Information Transmission, Russian Academy of Sciences

E-mails: vbel1@ime.usp.br, pablo@ime.usp.br, menchik@ime.usp.br,popov@ime.usp.br

\begin{abstract}
We consider a one-dimensional nearest-neighbor interacting particle system, which is a mixture of the simple exclusion process and the voter model. The state space is taken to be the countable set of the configurations that have a finite number of particles to the right of the origin and a finite number of empty sites to the left of it. We obtain criteria for the ergodicity and some other properties of this system using the method of Lyapunov functions.
\end{abstract}

Keywords: Exclusion Process, Lyapunov function, Voter Model

AMS Classification: $60 \mathrm{~K} 35,82 \mathrm{C}$

Running title: A mixture of Exclusion Process and Voter Model

\section{Introduction}

In this paper we consider a process that is a mixture of two nearest-neighbor one-dimensional interacting particle systems: the simple exclusion process and the voter model. Let us first define these two processes 
Definition 1.1 For $\eta \in\{0,1\}^{\mathbb{Z}}$ denote

$$
\eta_{x, y}(z)= \begin{cases}\eta(y), & \text { if } z=x, \\ \eta(x), & \text { if } z=y, \\ \eta(z), & \text { if } z \neq x, y\end{cases}
$$

and

$$
\eta_{x}(z)= \begin{cases}1-\eta(z), & \text { if } z=x, \\ \eta(z), & \text { if } z \neq x .\end{cases}
$$

A Markov process $\eta_{t} \in\{0,1\}^{\mathbb{Z}}, t \in[0,+\infty)$ is called

- Simple exclusion process with parameter $0 \leq p \leq 1$, if its generator $\Omega_{p}^{e}$ has the following form:

$$
\Omega_{p}^{e} f(\eta)=\sum_{x, y} p(x, y) \eta(x)(1-\eta(y))\left[f\left(\eta_{x, y}\right)-f(\eta)\right],
$$

where

$$
p(x, y)= \begin{cases}p, & \text { if } y=x-1 \\ 1-p, & \text { if } y=x+1 \\ 0, & \text { otherwise }\end{cases}
$$

- voter model, if its generator $\Omega^{v}$ is defined in the following way:

$$
\Omega^{v} f(\eta)=\sum_{x} c(x, \eta)\left[f\left(\eta_{x}\right)-f(\eta)\right]
$$

where

$$
c(x, \eta)= \begin{cases}\frac{1}{2}(\eta(x-1)+\eta(x+1)), & \text { if } \eta(x)=0, \\ \frac{1}{2}(2-\eta(x-1)-\eta(x+1)), & \text { if } \eta(x)=1 .\end{cases}
$$

The construction of those processes from their generators may be found in the book of Liggett (1985); see the first chapter and the beginning of the chapters corresponding to those processes. Harris graphical construction (see Durrett $(1988,1995)$ for instance) is an alternative approach to define these processes. It will be briefly reviewed and used in Section 2.

Let us call $\eta \in\{0,1\}^{\mathbb{Z}}$ a configuration of particles and let us interpret $\eta(x)=1$ as the presence of a particle at the site $x \in \mathbb{Z}$ in the configuration $\eta$ 
and $\eta(x)=0$ as the absence of it. The dynamics of both processes may be interpreted in terms of particles that hop on $\mathbb{Z}$ (the case of the exclusion process) or appear and disappear at the sites of $\mathbb{Z}$ (the case of the voter model).

In the exclusion process, there may be at most one particle at each site of $\mathbb{Z}$. If there is a particle at site $x$ and no particle at site $x+1$ (respectively at site $x-1)$, then the particle at $x$ jumps with rate $(1-p)$ (respectively $p$ ) to site $x+1$ (respectively $x-1$ ). This is a conservative dynamics, in the sense that neither particles are created nor disappear. Liggett (1976) described the set of invariant measures for this process. If $p=1 / 2$, the invariant measures are convex combinations of the translation invariant product measures parameterized with the density of particles. If $p>1 / 2$, the set of invariant measures contains also measures with support in the countable state space

$\mathcal{D}:=$ the set of configurations with a finite number of empty sites to the left of the origin and a finite number of particles to the right of it.

These measures are called blocking measures because, due to the exclusion rule and the accumulation of particles to the left of the origin, the flux of particles is null. Of course there are also blocking measures for $p<1 / 2$; they are obtained by the reflection $(\mathbb{Z} \rightarrow-\mathbb{Z})$ of those mentioned above. When an asymmetric exclusion process $(p \neq 1 / 2)$ is considered from a random position determined by a so-called second-class particle, a new set of invariant measures arises. They are called shock measures, they have support on configurations with different asymptotic densities to the left and right of the origin. The respective results have the origin in the works of Ferrari et al. (1991) and Ferrari (1992). See the review paper of Ferrari (1994) and the book of Liggett (1999) for an account of properties of these measures and the asymptotic behavior of the second class particle. Derrida et al. (1998) propose a nice alternative descriptions of shock measures for this process.

In the voter model, there may be at most one particle per site, however its dynamics is nonconservative: a new particle is born at an empty site $x$ at a rate proportional to the number of nearest neighbors of $x$ occupied by particles; and a particle that is present at a site $x$ disappears at a rate proportional to the number of the empty neighbors of $x$. Since only one site changes its value at any given time, this model is a particular case of the so called spin-flip models. There are only two invariant measures for the one-dimensional voter model defined above: one has the support on the configuration "all zeros" and the other one has the support on the configuration 
"all ones". The basic tool to prove those results is duality, a technique that allows to express properties of the voter model as properties of a dual process, a process obtained when one "looks backwards in time". There are two dual processes for the voter model: coalescing random walks and annihilating random walks. See Liggett (1985, Chapter V), Durrett (1995) for accounts on these and many other properties of the voter model.

If the voter model starts from the Heaviside configuration $\eta^{0}$, defined by $\eta^{0}(x)=\mathbf{1}_{\{x \leq 0\}}$, then at any future time it is a random translation of $\eta^{0}$. Indeed, the position of the rightmost particle $X_{t}=\max \left\{x: \eta_{t}(x)=1\right\}$ performs a nearest neighbor symmetric random walk and $\theta_{X_{t}} \eta_{t}=\eta_{0}$, where $\theta_{x}$ is translation by $x$. This example motivates the introduction of an equivalence relation: we say that two configurations $\eta$ and $\eta^{\prime}$ are equivalent and write $\eta \sim \eta^{\prime}$ if one of them is a translation of the other: there exists a $y \in \mathbb{Z}$ such that $\eta(x)=\eta^{\prime}(x+y)$ for all $x \in \mathbb{Z}$. Let $\tilde{\mathcal{D}}:=\mathcal{D} / \sim$ denote the set of equivalence classes induced by $\sim$. Let then $\mathcal{D}_{0}$ denote the set of the configurations in the equivalence class of $\eta^{0}$. In the voter model, $\eta_{0} \in \mathcal{D}_{0}$ implies $\eta_{t} \in \mathcal{D}_{0}$ for all $t$. Hence, denoting $\tilde{\eta}_{t}$ the equivalence class of $\eta_{t}$, we have that $\tilde{\eta}_{0}=\tilde{\eta}^{0}$ implies $\tilde{\eta}_{t} \equiv \tilde{\eta}^{0}$ (nothing moves). The process $\tilde{\eta}_{t} \in \tilde{\mathcal{D}}$ just defined, is isomorphic to $\theta_{X_{t}} \eta_{t}$, the voter model as seen from its rightmost particle.

Cox and Durrett (1995) studied one dimensional voter models on $\tilde{\mathcal{D}}$ with rate function $\sum_{y} q(|x-y|)|\eta(x)-\eta(y)|$ for some probability function $q(x)$. They show that if $\sum_{x}|x|^{3} q(x)<\infty$, then the process as seen from the rightmost particle $\tilde{\eta}_{t} \in \mathcal{D}$ is positive recurrent and hence admits a unique invariant (shock) measure. Calling $Y_{t}$ the leftmost hole, this implies that under the invariant measure the size of the hybrid zone - the region of coexistence of zeros and ones - $X_{t}-Y_{t}$ is bigger than -1 and finite with probability one; and of course its distribution is independent of $t$. They also prove that the expected value of $X_{t}-Y_{t}$ under the invariant measure is infinite and that $X_{t} / \sqrt{t}$ converges as $t \rightarrow \infty$ to a centered normal distribution with finite variance. The approach is based on a fine analysis of the (dual) process coalescing random walks. It is also shown there that there are no "stable" hybrid zones in dimension $d=2$ : if one starts with ones in the negative $x$ semiplane and zeros in the positive semiplane and paints 1s white and 0s black, then the normal distribution with variance $\sim t$ predicts the shade of grey we see at time $t$ in the horizontal direction.

Ferrari (1996) shows the existence of an invariant shock measure for the biased voter model as seen from the rightmost particle. In this model the 
rate function is given by $c_{2}(x, \eta)=(a \eta(x)+b(1-\eta(x))) c(x, \eta)$, with $c(x, \eta)$ as defined in (1.1). The proof in this case is more direct because it is based on straightforward dominations by supermartigales.

The goal of this paper is the study of the existence of shock measures in a mixture of the exclusion process and the voter model.

Definition 1.2 Let $\beta \in[0,1]$. A Markov process $\eta_{t} \in\{0,1\}^{\mathbb{Z}}, t \in[0,+\infty)$ is called hybrid process with mixing parameter $\beta$ and exclusion parameter $p$, if its generator is

$$
\Omega_{\beta, p}^{h}:=(1-\beta) \Omega_{p}^{e}+\beta \Omega^{v}
$$

The hybrid process $\tilde{\eta}_{t}$ (the class of equivalence of $\eta_{t}$ with initial configuration in $\mathcal{D}$ ) is a Markov process on $\tilde{\mathcal{D}}$. This process is a particular case of a model of random grammars, considered by Malyshev (1998). Models consisting of a mixture of a spin-flip dynamics and a symmetric exclusion dynamics are usually called in the literature "diffusion-reaction processes". When $\beta \gg 0$, an appropriate space-time rescaling with $\beta$ produces hydrodynamic limits rising the reaction-diffusion equation $\frac{\partial u}{\partial t}=\frac{\partial^{2} u}{\partial^{2} x}+f(u)$, whereas the function $f$ is related to the spin-flip dynamics and $u=u(x, t) \in[0,1], x, t \in \mathbb{R}_{+}$ corresponds to the macroscopic density of particles (De Masi et al. (1986)). In some cases these equations accept traveling-wave solutions - solutions of the type $u(x, t)=u_{0}(x-v t)$ for some speed $v$ with $\lim _{x \rightarrow \infty} u_{0}(x)=0$, $\lim _{x \rightarrow-\infty} u_{0}(x)=1$. This motivates the question about the existence of a microscopic counterpart of the macroscopic traveling wave solutions. A particular case of reaction process is the growth model, a process with rate function $c(x, \eta)(1-\eta(x))$, where $c(x, \eta)$ has been defined in (1.1): 0 flips to 1 at rate proportional to the number of ones in the neighborhood, but 1 never flips to 0. Bramson et al. (1986) showed the existence of an invariant (shock) measure for the process $\tilde{\eta}_{t}$, where $\eta_{t}$ is any nontrivial mixture of the exclusion process and the growth model. Cammarota and Ferrari (1991) proved the Normal asymptotic behavior of $\left(X_{t}-\mathbf{E} X_{t}\right) / \sqrt{t}$ for this mixture. Machado (1998) studied this process in a strip and in $\mathbb{Z}^{d}$.

Let $\tilde{\tau}_{c}(\tilde{\eta})$ be the first time the process $\tilde{\eta}_{t}$ starting with the configuration $\tilde{\eta} \in \tilde{\mathcal{D}}$ hits $\tilde{\eta}^{0}$, the Heaviside configuration defined above. The subscript $c$ refers to continuous time (as a counterpart of a discrete-time process to be introduced below). Let us recall some classical definitions. We say that the process $\tilde{\eta}_{t}$ is transient, if $\mathbf{P}\left(\tilde{\tau}_{c}(\tilde{\eta})<\infty\right)<1$ and recurrent, if $\mathbf{P}\left(\tilde{\tau}_{c}(\tilde{\eta})<\infty\right)=$ 1 . In the last case we say that the process is positive recurrent if $\mathbf{E}\left(\tilde{\tau}_{c}(\tilde{\eta})\right)<\infty$ 
and null recurrent if this expectation is infinity. An irreducible countable Markov chain is ergodic if it has a unique invariant measure. Since, except for the pure voter model, $\tilde{\eta}_{t}$ is irreducible, positive recurrence is equivalent to ergodicity in our context. The following theorem contains our results.

Theorem 1.1 Let $\eta_{t}$ be a process in $\mathcal{D}$ with generator $\Omega_{\beta, p}^{h}$. Let $\tilde{\eta}_{t}$ be the corresponding process in the space of classes of equivalence $\tilde{\mathcal{D}}$.

1. Exclusion process. Assume $\beta=0$. Then the process $\tilde{\eta}_{t}$ is ergodic for $p>1 / 2$ and transient for $p \leq 1 / 2$.

2. Hybrid process. Assume $0<\beta<1$. Then

i) There exists $\beta_{c}<1$ such that for any $\beta>\beta_{c}$ and any $p \in(0,1)$ the process $\tilde{\eta}_{t}$ is ergodic.

ii) For any $p \geq 1 / 2$ and any $\beta$, the process is ergodic.

3. Voter model. Assume $\beta=1$. Then the process $\tilde{\eta}_{t}$ is positive recurrent. Moreover, for any initial configuration $\tilde{\eta} \in \tilde{\mathcal{D}}$ and any $\varepsilon>0$,

$$
\mathbf{E}\left(\tilde{\tau}_{c}(\tilde{\eta})\right)^{3 / 2-\varepsilon}<\infty ; \quad \mathbf{E}\left(\tilde{\tau}_{c}(\tilde{\eta})\right)^{3 / 2+\varepsilon}=\infty .
$$

The fact that the exclusion process $\tilde{\eta}_{t}$ in $\tilde{\mathcal{D}}$ is ergodic for $p>1 / 2$ follows immediately from well known results of Liggett $(1976,1985)$ who described the invariant measures for $\eta_{t}$ in the irreducible classes of $\mathcal{D}$. Since the system is conservative, ergodicity of $\eta_{t}$ on any irreducible class of $\mathcal{D}$ is equivalent to ergodicity of $\tilde{\eta}_{t}$ on $\tilde{\mathcal{D}}$. Our alternative approach does not use the knowledge of the invariant measure. When $p \leq 1 / 2$, the results of Liggett imply only that the process $\eta_{t}$ is not positive recurrent; our result says that it is transient. For $p<1 / 2$, the transience holds immediately from laws of large numbers for the leftmost hole and the rightmost particle. For $p=1 / 2$, the transience is a more delicate matter.

The bounds in (1.3) show the velocity of the convergence of the voter model to the invariant measure, which is the singleton supported by $\mathcal{D}_{0}$. It may be the case that these bounds could be obtained from the duality of the voter model to the coalescing random walks, however, we have not investigated this approach.

Our main results are the conditions for ergodicity for the hybrid model described in point (2) of the theorem. It says that if either the proportion 
of voter in the hybrid process is large enough or the exclusion process has no drift to the right, then the hybrid process is ergodic. Item (1) says that exclusion is transient for $p \leq 1 / 2$, while item (3) says that voter is always positive recurrent. The first part of item (2) says that voter "wins" if the proportion of voter is sufficiently large, uniformly on the exclusion asymmetry; the relevant point in the second part of item (2) says that for the symmetric exclusion, any proportion of voter guarantees ergodicity.

We are not totally satisfied with this result because sufficient conditions for transience are missing. One would like to show that if the asymmetry of the exclusion process has a tendency to "escape" from $\mathcal{D}$ then an addition of a small proportion of the voter model will not be able to prevent it from escaping. But for now, it is still very unclear to us, if the process could be transient in this case. We state now a conjecture for the nonergodicity of the hybrid process. A heuristic argument supporting the conjecture is presented in Section 0 .

Conjecture 1.1 For any $p<1 / 2$ there exists a $\beta_{0}(p)>0$ such that for any $\beta<\beta_{0}(p)$, the hybrid process $\tilde{\eta}_{t}$ with parameters $\beta$ and $p$ is not ergodic.

The parameter space $\{(p, \beta): p, \beta \in[0,1]\}$ is partitioned in three regions: ergodicity, transience and null-recurrence. Presumably the region of transience satisfies the property: if the hybrid process with parameters $\left(p_{0}, \beta_{0}\right)$ is transient, then the one with parameters $\left(p_{1}, \beta_{1}\right)$ will be also transient for $p_{1} \leq p_{0}$ and $\beta_{1} \leq \beta_{0}$. But we do not have any monotonicity argument at hand to argue this. We know that the transience region is nonempty because it contains the segment $[0,1 / 2] \times\{0\}$, but we do not know how to prove that it contains points in the interior of the parameter space.

How stable under changes of the dynamics are our results? Can we extend Theorem 1.1 to nonnearest-neighbors processes? When $\beta=1$, only voter, the answer is given by Cox and Durrett (1995), as described above. When $\beta=0$, only exclusion, it is known that the process is not ergodic on $\mathcal{D}$ if $p(x, y)$ is symmetric (all invariant measures are translation invariant in this case), but it is an open problem of Liggett (1985, Section VIII.7, Problem 6) in the case when $p(x, y)$ is asymmetric. The conjecture is that if $p(x, y)=$ $q(y-x)$, for some $q$, then the system would be ergodic under the condition $\sum_{x} x q(x)<0$. In the final remarks we explain where our approach fails to work when extended to the nonnearest-neighbors case.

Motivations coming from real life, description of shock measures in other one-dimensional models and nice conjectures about the existence of shock 
measures in other systems can be found in the introduction of Cox and Durrett (1995).

Theorem 1.1 is proven for the discrete-time version of $\tilde{\eta}_{t}$ and then standard arguments are used to prove the continuous counterpart. The discrete process is a Markov chain in $\tilde{\mathcal{D}}$. The basic tool is a set of theorems from Fayolle et al. (1995), which give conditions for ergodicity, recurrence and transience of denumerable Markov chains using so-called Lyapunov functions. The application of these functions to the processes in interest produces sub or super martingales, which can be used straightforwardly to show the desired properties. The problem is that these functions are frequently hard to find. One of the contributions of this paper is the exhibition of Lyapunov functions that work for the exclusion process, the voter model and their mixture.

The paper is organized in the following manner. In Section 2 we introduce the discrete version of the process $\tilde{\eta}_{t}$. In Section 3 we state the results of Fayolle et al. (1995) we need. In Section 1 we introduce the Lyapunov functions of the process that will be relevant in the proofs. In Sections 5 , 6 and 17 we state and prove the results for the discrete-time versions of the exclusion process, the voter model and the hybrid process respectively. In Section 8 we show how to pass from the discrete to the continuous time and prove Theorem 1.1.

\section{Discrete and continuous-time processes}

In this section we introduce discrete-time versions of the exclusion process, voter model, and their mixture that have been defined in the previous section, and establish their relations with the continuous-time processes.

Let $\eta$ be a configuration from $\{0,1\}^{\mathbb{Z}}$. We say that a discrepancy of type 01 (10) occurs in $\eta$ at the site $x$, if $\eta(x-1)=0, \eta(x)=1$ (resp., $\eta(x)=1, \eta(x+1)=0)$. The above defined countable set $\mathcal{D}$ is the set of those configurations of $\{0,1\}^{\mathbb{Z}}$ in which there is only a finite number of discrepancies, and the number of discrepancies of type 10 minus the number of discrepancies of type 01 is equal to 1 . Then it is easy to see that

$$
\begin{aligned}
\mathcal{D}=\left\{\eta \in\{0,1\}^{\mathbb{Z}}: \text { there exist } i_{0}, j_{0}\right. \text { such that } \\
\\
\left.\quad \eta(i)=1 \text { for } i \leq i_{0} \text { and } \eta(j)=0 \text { for } j \geq j_{0}\right\}
\end{aligned}
$$

and that $\mathcal{D}$ is countable. 
The discrete time exclusion process with parameter $p$ (to be called here $\mathrm{EP}(p))$ is a Markov process with the state space $\mathcal{D}$ and the following dynamics: for every $n \geq 0$, if $\eta$ is the state at time $n$ then $\eta^{\prime}$, the state at time $n+1$, is obtained by the following procedure (i)-(ii):

(i) we choose one of the discrepancies of $\eta$ with uniform distribution; say the discrepancy at the site $x$ has been chosen, then

(ii) if the discrepancy is 01 (10) then we exchange 0 and 1 with the probability $0<p<1$ (resp., $0<q:=1-p<1$ ) while nothing is changed with the resting probability $q$ (resp., $1-q$ ).

The Exclusion Process just defined is a countable Markov chain on $\mathcal{D}$.

Let us define now the discrete time Voter Model (to be called VM) and the discrete time hybrid process (to be called $\operatorname{HP}(\beta, p)$, where $\beta$ is the mixing parameter and $p$ is the exclusion parameter). For VM the step (i) is the same, and (ii) is substituted by the following:

(ii') the chosen discrepancy is substituted by either 11 or 00 with probabilities $1 / 2$.

To construct $\operatorname{HP}(\beta, p)$, we first execute (i), and then with probability $1-\beta$ we execute (ii) (i.e. make a step of the exclusion process), and with probability $\beta$ we execute (ii') (i.e. make a step of the voter model). We use the notation $\left(\xi_{n}: n \in \mathbb{N}\right)$ for the $\operatorname{HP}(\beta, p) . \xi_{n}$ denotes the configuration of the system at time $n$.

In (2.2) below we shall present the relation between the discrete-time hybrid process $\left(\xi_{n}: n \in \mathbb{N}\right)$ and the continuous-time hybrid process $\left(\eta_{t}\right.$ : $t \geq 0$ ) with the mixing parameter $\beta$ and the exclusion parameter $p$. To this end, we shall need the Harris graphical construction for $\left(\eta_{t}: t \geq 0\right)$, which we now briefly recall. It is a "superposition" of the graphical construction for the voter model (see Durrett (1995)) with that for the exclusion process (see Ferrari (1992)) with the respective weights $\beta$ and $(1-\beta)$.

Let $\left\{\left(\mathcal{N}_{t}^{x, x+1}, t \geq 0\right)\right\}_{x \in \mathbb{Z}},\left\{\left(\mathcal{N}_{t}^{x, x-1}, t \geq 0\right)\right\}_{x \in \mathbb{Z}},\left\{\left(\mathcal{M}_{t}^{x, x+1}, t \geq 0\right)\right\}_{x \in \mathbb{Z}}$, $\left\{\left(\mathcal{M}_{t}^{x, x-1}, t \geq 0\right)\right\}_{x \in \mathbb{Z}}$ be four independent families of Poisson point processes with the respective rates $(1-\beta) p,(1-\beta) q, \beta / 2$ and $\beta / 2$. Given the initial configuration $\eta_{0}$, the dynamics of the process $\eta_{t}, t \geq 0$ is determined by those Poisson processes in the following manner. If there is a Poisson event at time $t$ in $\mathcal{N}^{x, x+1}$ (resp., $\mathcal{N}^{x, x-1}$ ), which means $\mathcal{N}_{t}^{x, x+1}-\mathcal{N}_{t^{-}}^{x, x+1}=1$, and if $x$ has a particle while $x+1$ is empty (resp., $x-1$ is empty) in $\eta_{t^{-}}$, then the 
particle jumps from $x$ to $x+1$ (resp., $x-1$ ) at time $t$. If there is a Poisson event at time $t$ in $\mathcal{M}^{x, x+1}$ (resp., $\mathcal{M}^{x, x-1}$ ), then the site $x+1$ (resp., $x-1$ ) acquires the same state at time $t$ as the state of $x$ in $\eta_{t^{-}}$.

Let $\tau_{0}=0$ and for $n \geq 1$, set

$$
\begin{aligned}
& \tau_{n}=\inf \left\{t>\tau_{n-1}\right.: \sum_{x, y:|x-y|=1}\left|\eta_{t^{-}}(x)-\eta_{t^{-}}(y)\right| \\
&\left.\times\left(\mathcal{N}^{x, y}\left(\tau_{n-1}, t\right]+\mathcal{M}^{x, y}\left(\tau_{n-1}, t\right]\right)>0\right\}
\end{aligned}
$$

where $\mathcal{N}(s, t]$ denotes the number of the Poisson events in the time interval $(s, t]$ for the process $\mathcal{N}$. We call $\tau_{n}$ the instants of attempted jumps of the process $\eta_{t}$. It follows then from our definitions that if $\eta_{0}=\xi_{0}$, then

$$
\left(\xi_{n}: n \geq 0\right)=\left(\eta_{\tau_{n}}: n \geq 0\right) \quad \text { in distribution. }
$$

\section{Criteria for recurrence and transience of Markov chains}

In this section we state the criteria for ergodicity, recurrence and transience of countable Markov chains to be used in the sequel. The next four theorems are Theorems 2.2.3, 2.2.1, 2.2.2, 2.2.7, respectively, of Fayolle et al. (1995).

Theorem 3.1 Let $\xi_{t}, t=0,1,2, \ldots$ be an irreducible Markov chain with the countable state space $X$. Suppose that there exist a positive function $f(x)$ and a finite set $A \subset X$ such that

$$
\mathbf{E}\left(f\left(\xi_{t+1}\right)-f\left(\xi_{t}\right) \mid \xi_{t}=x\right) \leq-\varepsilon
$$

for some $\varepsilon>0$ and all $x \in X \backslash A$, and that

$$
\mathbf{E}\left(f\left(\xi_{t+1}\right) \mid \xi_{t}=x\right)<\infty
$$

for $x \in A$. Then the Markov chain is ergodic.

Theorem 3.2 Let $\xi_{t}, t=0,1,2, \ldots$ be an irreducible Markov chain with the countable state space $X$. Suppose that there exist a positive function $f(x)$, $f(x) \rightarrow \infty$ as $x \rightarrow \infty$, and a finite set $A \subset X$ such that

$$
\mathbf{E}\left(f\left(\xi_{t+1}\right)-f\left(\xi_{t}\right) \mid \xi_{t}=x\right) \leq 0
$$

for all $x \in X \backslash A$. Then the Markov chain is recurrent. 
Theorem 3.3 Let $\xi_{t}, t=0,1,2, \ldots$ be an irreducible Markov chain with the countable state space $X$. Suppose that there exist a positive function $f(x)$ and a set $A \subset X$ such that (3.3) holds for all $x \in X \backslash A$ and

$$
f\left(x_{0}\right)<\inf _{x \in A} f(x)
$$

for some $x_{0} \notin A$. Then the Markov chain is transient.

Theorem 3.4 Let $\xi_{t}, t=0,1,2, \ldots$ be an irreducible Markov chain with the countable state space $X$. Suppose that there exist a positive function $f(x)$ and a constant $C$ such that if $f(x)>C$, then

$$
\mathbf{E}\left(f\left(\xi_{t+1}\right)-f\left(\xi_{t}\right) \mid \xi_{t}=x\right) \geq \varepsilon
$$

for some $\varepsilon>0$, and suppose that for some $K>0$

$$
\left|f\left(\xi_{t+1}\right)-f\left(\xi_{t}\right)\right| \leq K \quad \text { a.s. }
$$

Then the Markov chain is transient.

Besides the ergodicity, we are going to study the existence of moments of the hitting time of the set $\mathcal{D}_{0}$. To do this, we shall need the following result of Aspandiiarov et al. (1996, Theorem 1)

Theorem 3.5 Let $A$ be some positive real number. Suppose that we are given $a\left\{\mathcal{F}_{n}\right\}$-adapted stochastic process $X_{n}, n \geq 0$, taking values in an unbounded subset of $\mathbb{R}_{+}$. Denote by $\tau_{A}$ the moment when the process $X_{n}$ enters the set $(0, A)$. Assume that there exist $\lambda>0, p_{0} \geq 1$ such that for any $n, X_{n}^{2 p_{0}}$ is integrable and

$$
\mathbf{E}\left(X_{n+1}^{2 p_{0}}-X_{n}^{2 p_{0}} \mid \mathcal{F}_{n}\right) \leq \lambda X_{n}^{2 p_{0}-2}
$$

on $\left\{\tau_{A}>n\right\}$. Then there exists a positive constant $C=C\left(\lambda, p_{0}\right)$ such that for all $x \geq 0$ whenever $X_{0}=x$ with probability 1

$$
\mathbf{E} \tau_{A}^{p_{0}} \leq C x^{2 p_{0}} .
$$




\section{Functions of the process}

For the sake of brevity we will substitute in the sequel the expression "block of zeros" by "0-block" and "block of ones" by "1-block".

A class of equivalence $S \in \tilde{\mathcal{D}}$ can be identified by a finite set of positive numbers in the following form:

$$
S=\ldots 111 \overbrace{0000}^{n_{1}} \overbrace{11111}^{m_{1}} \overbrace{0000}^{n_{2}} \overbrace{11111}^{m_{2}} \ldots \overbrace{00000}^{n_{N}} \overbrace{1111}^{m_{N}} 000 \ldots,
$$

where $n_{i}=n_{i}(S)$ is the size of $i$-th 0 -block, $m_{i}=m_{i}(S)$ is the size of $i$-th 1 block, $N=N(S)$ is the number of 1-blocks not including the leftmost infinite 1-block. In the sequel the word "configuration" will usually mean "class of equivalence". So, for $S \in \tilde{\mathcal{D}}$ we can simply write $S=\left(n_{1}, m_{1}, \ldots, n_{N}, m_{N}\right)$.

Denote $r_{0}=0, r_{i}=\sum_{j=1}^{i}\left(m_{j}+n_{j}\right), l_{i}=\sum_{j=1}^{i-1}\left(m_{j}+n_{j}\right)+n_{i}+1$, $i=1, \ldots, N$. Let $\eta$ be the configuration from the class of equivalence $S$ such that $\eta(x)=1$ for $x \leq 0$ and $\eta(1)=0$. Define the configurations $\eta_{k}, \eta_{k}^{\leftarrow}, \eta_{k}^{+r}$, $\eta_{k}^{+l}, \eta_{k}^{-r}, \eta_{k}^{-l}$ in the following way:

- $\eta_{k}(x)=\eta(x)$ for $x \neq r_{k}, r_{k}+1, \eta_{k}\left(r_{k}\right)=0, \eta_{k}\left(r_{k}+1\right)=1, k=$ $0, \ldots, N$;

- $\eta_{k}^{\overleftarrow{k}}(x)=\eta(x)$ for $x \neq l_{k}, l_{k}-1, \eta_{k}^{\leftarrow}\left(l_{k}\right)=0, \eta_{k}^{\overleftarrow{k}}\left(l_{k}-1\right)=1, k=1, \ldots, N$

- $\eta_{k}^{+r}(x)=\eta(x)$ for $x \neq r_{k}+1, \eta_{k}^{+r}\left(r_{k}+1\right)=1, k=0, \ldots, N$;

- $\eta_{k}^{+l}(x)=\eta(x)$ for $x \neq l_{k}-1, \eta_{k}^{+l}\left(l_{k}-1\right)=1, k=1, \ldots, N$;

- $\eta_{k}^{-r}(x)=\eta(x)$ for $x \neq r_{k}, \eta_{k}^{-r}\left(r_{k}\right)=0, k=0, \ldots, N$;

- $\eta_{k}^{-l}(x)=\eta(x)$ for $x \neq l_{k}, \eta_{k}^{-l}\left(l_{k}\right)=0, k=1, \ldots, N$.

and $S_{k}^{\rightarrow}, S_{k}^{\leftarrow}, S_{k}^{+r}, S_{k}^{+l}, S_{k}^{-r}, S_{k}^{-l}$ are the corresponding classes of equivalence. Informally speaking,

- $S_{k} \rightarrow$ is the configuration obtained from $S$ by moving the rightmost 1 of the $k$-th 1 -block by 1 unit to the right, $k=0, \ldots, N$;

- $S_{k}^{\leftarrow}$ is the configuration obtained from $S$ by moving the leftmost 1 of the $k$-th 1 -block by 1 unit to the left, $k=1, \ldots, N$;

- $S_{k}^{+r}$ is the configuration obtained from $S$ by adding an extra 1 to the right of the $k$-th 1 -block, $k=0, \ldots, N$; 
- $S_{k}^{+l}$ is the configuration obtained from $S$ by adding an extra 1 to the left of the $k$-th 1-block, $k=1, \ldots, N$;

- $S_{k}^{-r}$ is the configuration obtained from $S$ by removing the rightmost 1 from the $k$-th 1-block, $k=0, \ldots, N$;

- $S_{k}^{-l}$ is the configuration obtained from $S$ by removing the leftmost 1 from the $k$-th 1-block, $k=1, \ldots, N$.

Clearly, EP can transform $S$ to $S_{k}^{\rightarrow}$ or $S_{k}^{\leftarrow}$, while using VM we can get $S_{k}^{ \pm r}$ or $S_{k}^{ \pm l}$.

Denote also $R_{i}=\sum_{j=1}^{i} n_{j}, T_{i}=\sum_{j=i}^{N} m_{j}$, and let

$$
|S|=\sum_{j=1}^{N}\left(m_{j}+n_{j}\right)=R_{N}+T_{1}
$$

stand for the length of "nontrivial" part of configuration $S$. Notational convention: $R_{0}=T_{N+1}=0$.

We define two functions $f_{1}, f_{2}: \tilde{\mathcal{D}} \mapsto \mathbb{R}$, which will play the crucial role in our arguments:

$$
\begin{aligned}
f_{1}(S) & =\frac{1}{2}\left(\sum_{k: S(k)=1}\left(\sum_{m<k} \mathbf{1}_{\{S(m)=0\}}\right)+\sum_{k: S(k)=0}\left(\sum_{m>k} \mathbf{1}_{\{S(m)=1\}}\right)\right) \\
& =\frac{1}{2}\left(\sum_{i=1}^{N} m_{i} R_{i}+\sum_{i=1}^{N} n_{i} T_{i}\right) \\
& =\sum_{i=1}^{N} m_{i} R_{i}=\sum_{i=1}^{N} n_{i} T_{i},
\end{aligned}
$$

and

$$
\begin{aligned}
f_{2}(S) & =\frac{1}{2}\left(\sum_{k: S(k)=1}\left(\sum_{m<k} \mathbf{1}_{\{S(m)=0\}}\right)^{2}+\sum_{k: S(k)=0}\left(\sum_{m>k} \mathbf{1}_{\{S(m)=1\}}\right)^{2}\right) \\
& =\frac{1}{2}\left(\sum_{i=1}^{N} m_{i} R_{i}^{2}+\sum_{i=1}^{N} n_{i} T_{i}^{2}\right),
\end{aligned}
$$

for all $S \in \tilde{\mathcal{D}}$. 
Before going further, let us make some remarks about $f_{1}, f_{2}$. The value $f_{1}(S)$ is equal exactly to the number of nearest-neighbor transpositions needed to pass from $S$ to $\mathcal{D}_{0}$, that is, $f_{1}(S)$ is in some sense the "distance" from $S$ to the trivial configuration. Unfortunately, as we will see later, the function $f_{1}$ does not "work" well for some configurations $S$ (namely, for $S$ such that $N(S)$ is small with respect to $|S|)$. The function $f_{2}$ is the result of our attempts to modify $f_{1}$ in order to eliminate this disadvantage; we cannot give any intuitive meaning of $f_{2}(S)$.

Let us obtain some relations between $|S|, f_{1}(S)$ and $f_{2}(S)$.

Lemma 4.1 For any $S \in \mathcal{D}$ the following holds:

i) $|S| / 2 \leq f_{1}(S) \leq|S|^{2} / 4$;

ii) $|S|^{2} / 4 \leq f_{2}(S) \leq|S|^{3} / 8$;

iii) $f_{1}(S) \leq\left(f_{2}(S)\right)^{3 / 4}$.

Proof. The proof of i)-ii) is simple. We have

$$
\begin{gathered}
f_{1}(S)=\frac{1}{2}\left(\sum_{i=1}^{N} m_{i} R_{i}+\sum_{i=1}^{N} n_{i} T_{i}\right) \geq \frac{1}{2}\left(R_{N}+T_{1}\right)=\frac{|S|}{2}, \\
f_{1}(S)=\sum_{i=1}^{N} m_{i} R_{i} \leq R_{N} \sum_{i=1}^{N} m_{i}=R_{N} T_{1} \leq \frac{\left(R_{N}+T_{1}\right)^{2}}{4}=\frac{|S|^{2}}{4},
\end{gathered}
$$

and, analogously,

$$
\begin{gathered}
f_{2}(S) \geq \frac{1}{2}\left(R_{N}^{2}+T_{1}^{2}\right) \geq \frac{1}{4}\left(R_{N}+T_{1}\right)^{2}=\frac{|S|^{2}}{4} \\
f_{2}(S) \leq \frac{1}{2}\left(R_{N}^{2} \sum_{i=1}^{N} m_{i}+T_{1}^{2} \sum_{i=1}^{N} n_{i}\right)=\frac{1}{2} R_{N} T_{1}\left(R_{N}+T_{1}\right) \leq \frac{|S|^{3}}{8} .
\end{gathered}
$$

Let us prove iii). We shall make use of the following simple consequence of the Jensen inequality: if we have $n$ positive numbers $\gamma_{1}, \ldots, \gamma_{n}$ such that $\sum_{i=1}^{n} \gamma_{i}=1$, then for any $x_{1}, \ldots, x_{n}$

$$
\gamma_{1} x_{1}+\cdots+\gamma_{n} x_{n} \leq\left(\gamma_{1} x_{1}^{2}+\cdots+\gamma_{n} x_{n}^{2}\right)^{1 / 2}
$$


Denote $\alpha_{i}=m_{i} /|S|, \beta_{i}=n_{i} /|S|$, so $\sum_{i=1}^{N}\left(\alpha_{i}+\beta_{i}\right)=1$. Using (4.2) and ii), we get

$$
\begin{aligned}
f_{1}(S) & =\frac{1}{2} \sum_{i=1}^{N}\left(m_{i} R_{i}+n_{i} T_{i}\right)=\frac{|S|}{2} \sum_{i=1}^{N}\left(\alpha_{i} R_{i}+\beta_{i} T_{i}\right) \\
& \leq \frac{|S|}{2}\left(\sum_{i=1}^{N}\left(\alpha_{i} R_{i}^{2}+\beta_{i} T_{i}^{2}\right)\right)^{1 / 2}=\frac{\sqrt{|S|}}{\sqrt{2}}\left(f_{2}(S)\right)^{1 / 2} \\
& \leq \frac{\sqrt{2}\left(f_{2}(S)\right)^{1 / 4}}{\sqrt{2}}\left(f_{2}(S)\right)^{1 / 2}=\left(f_{2}(S)\right)^{3 / 4}
\end{aligned}
$$

thus completing the proof of Lemma 4.1.

As usual, symbols $\mathbf{P}$ and $\mathbf{E}$ stand for probability and expectation. When using them may look ambiguous, we use symbol $\mathbf{E}_{p}^{e}\left(\mathbf{P}_{p}^{e}\right)$ to denote expectation (probability) w.r.t. $\operatorname{EP}(p), \mathbf{E}^{v}\left(\mathbf{P}^{v}\right)$ stands for expectation (probability) w.r.t. VM, $\mathbf{E}_{\beta, p}^{h}\left(\mathbf{P}_{\beta, p}^{h}\right)$ denotes expectation (probability) w.r.t. $\operatorname{HP}(\beta, p)$.

\section{$5 \quad$ Exclusion process}

In this section we shall study the EP using the method of Lyapunov functions.

Theorem 5.1 If $p>q$, then the exclusion process is ergodic.

Proof. As we noticed before, EP can transform a configuration $S$ only either to $S_{k} \rightarrow$ or to $S_{k}^{\leftarrow}$, where the notations $S_{k}$ and $S_{k}^{\leftarrow}$ have been introduced in Section 4 . Then, it is elementary to get that

$$
\begin{aligned}
f_{2}\left(S_{k}\right)-f_{2}(S) & =\frac{1}{2}\left(\left(R_{k}+1\right)^{2}-R_{k}^{2}+\left(T_{k+1}+1\right)^{2}-T_{k+1}^{2}\right) \\
& =1+R_{k}+T_{k+1}
\end{aligned}
$$

and

$$
\begin{aligned}
f_{2}\left(S_{k}^{\leftarrow}\right)-f_{2}(S) & =\frac{1}{2}\left(\left(R_{k}-1\right)^{2}-R_{k}^{2}+\left(T_{k}-1\right)^{2}-T_{k}^{2}\right) \\
& =1-R_{k}-T_{k}
\end{aligned}
$$

Combining (5.1) and (5.2), we have that 


$$
\mathbf{E}\left(f_{2}\left(\xi_{t+1}\right)-f_{2}\left(\xi_{t}\right) \mid \xi_{t}=S\right)=\frac{N+q}{2 N+1}-\frac{p-q}{2 N+1} \sum_{i=1}^{N}\left(R_{i}+T_{i}\right) .
$$

Since $R_{N}+T_{1}=|S|, R_{i} \geq i$ and $T_{i} \geq N-i+1$, it is straightforward to get that

$$
\sum_{i=1}^{N}\left(R_{i}+T_{i}\right) \geq \max \{|S|, N(N+1)\} .
$$

Using this fact, we get from (5.3) that for any $\varepsilon>0$

$$
\mathbf{E}\left(f_{2}\left(\xi_{t+1}\right)-f_{2}\left(\xi_{t}\right) \mid \xi_{t}=S\right)<-\varepsilon
$$

for all but finitely many $S$. So, by Theorem 3.1, $\operatorname{EP}(p)$ is ergodic when $p>1 / 2$.

Theorem 5.2 When $p \leq q$ the exclusion process is transient.

Proof. First we consider the case $p<q$.

With $S_{k}$ and $S_{k}^{\leftarrow}$ being as defined above, we have that

$$
f_{1}\left(S_{k}^{\leftarrow}\right)-f_{1}(S)=-1
$$

and

$$
f_{1}\left(S_{k}^{\rightarrow}\right)-f_{1}(S)=1,
$$

so that for some $\varepsilon=\varepsilon(p, q)>0$

$$
\mathbf{E}_{p}^{e}\left(f_{1}\left(\xi_{t+1}\right)-f_{1}\left(\xi_{t}\right) \mid \xi_{t}=S\right)=\frac{N(q-p)}{2 N+1}+\frac{q}{2 N+1} \geq \varepsilon
$$

and, clearly, $\left|f_{1}\left(\xi_{t+1}\right)-f_{1}\left(\xi_{t}\right)\right| \leq 1$ almost surely. Then by Theorem 3.4, the process $\xi_{t}$ is transient.

Let us turn now to the case $p=q=1 / 2$.

Using the function $f_{1}(S)$ defined above and (5.5), (5.6), we have that

$$
\mathbf{E}_{1 / 2}^{e}\left(f_{1}\left(\xi_{t+1}\right)-f_{1}\left(\xi_{t}\right) \mid \xi_{t}=S\right)=\frac{1}{2(2 N+1)}
$$

so Theorem 3.3 does not apply. Therefore, we need a different approach. 
We fix an arbitrary $\alpha>0$ and define the function $\psi: \mathcal{D} \backslash \mathcal{D}_{0} \mapsto \mathbb{R}$ by

$$
\psi(S):=\left(f_{1}(S)\right)^{-\alpha} \text {. }
$$

Note that the definition is correct because $f_{1}(S)>0$ for $S \notin \mathcal{D}_{0}$. (Actually, for the need of Theorem 5.2 it is sufficient to take $\alpha=1$, but, since we will need analogous calculations later in this paper, at this point we prefer to do the calculations for arbitrary $\alpha>0$.) To study the properties of the process $\psi\left(\xi_{t}\right)$, we need the following lemma.

Lemma 5.1 For any $C>0$ the set

$$
A_{C}=\left\{S: f_{1}(S)<C N(S)\right\}
$$

is finite.

Proof. Clearly, $R_{i} \geq i$ and $m_{i} \geq 1$, so $f_{1}(S) \geq N(S)(N(S)+1) / 2$. Thus, for a configuration $S$ to belong to $A_{C}$, it is necessary that the number of 1-blocks be less than $2 C-1$, so $A_{C}$ is a subset of

$$
\left\{S: f_{1}(S)<C(2 C-1)\right\}
$$

which is obviously finite.

It follows from (5.5) and (5.6) that

$$
\mathbf{E}_{1 / 2}^{e}\left(\left(f_{1}\left(\xi_{t+1}\right)-f_{1}\left(\xi_{t}\right)\right)^{2} \mid \xi_{t}=S\right)=\frac{1}{2}
$$

By elementary calculations, we get that for any $\alpha>0$ there exist two positive numbers $C_{1}=C_{1}(\alpha), C_{2}=C_{2}(\alpha)$ such that

$$
(x+1)^{-\alpha}-1 \leq-\alpha x+C_{1} x^{2},
$$

when $|x|<C_{2}$.

Using (5.8), (5.10), (5.11) and Lemma 5.1 we get

$$
\begin{aligned}
& \mathbf{E}_{1 / 2}^{e}\left(\psi\left(\xi_{t+1}\right)-\psi\left(\xi_{t}\right) \mid \xi_{t}=S\right) \\
& \quad=f_{1}^{-\alpha}(S) \mathbf{E}_{1 / 2}^{e}\left(\left(\frac{f_{1}\left(\xi_{t+1}\right)}{f_{1}\left(\xi_{t}\right)}\right)^{-\alpha}-1 \mid \xi_{t}=S\right) \\
& \quad=f_{1}^{-\alpha}(S) \mathbf{E}_{1 / 2}^{e}\left(\left(\frac{f_{1}\left(\xi_{t+1}\right)-f_{1}\left(\xi_{t}\right)}{f_{1}\left(\xi_{t}\right)}+1\right)^{-\alpha}-1 \mid \xi_{t}=S\right) \\
& \quad \leq f_{1}^{-\alpha}(S)\left(-\frac{\alpha}{f_{1}(S)} \cdot \frac{1}{2(2 N+1)}+\frac{C_{1}}{2 f_{1}^{2}(S)}\right) \\
& \quad=f_{1}^{-\alpha-2}(S)\left(-\frac{\alpha f_{1}(S)}{2(2 N+1)}+\frac{C_{1}}{2}\right)<0
\end{aligned}
$$


on $\left\{S: f_{1}(S)>\max \left\{1 / C_{2}, C_{1}(2 N(S)+1) / \alpha\right\}\right\}$, and hence for all but finitely many $S$. Applying Theorem 3.4, we finish the proof of Theorem 5.2 .

\section{$6 \quad$ Voter model}

The subject of this section is the discrete time voter model. For the process starting from a configuration $S$ denote by $\tau(S)$ the moment of hitting the set $\mathcal{D}_{0}$. The main result of this section is the following

Theorem 6.1 The discrete time voter model is positive recurrent. Moreover, for any initial configuration $S_{0}$ and any $\varepsilon>0$

$$
\mathbf{E}\left(\tau\left(S_{0}\right)\right)^{3 / 2-\varepsilon}<\infty
$$

and

$$
\mathbf{E}\left(\tau\left(S_{0}\right)\right)^{3 / 2+\varepsilon}=\infty .
$$

Proof. Since positive recurrence means just the existence of $\mathbf{E} \tau\left(S_{0}\right)$, we shall turn directly to the proof of (6.1). The idea is to apply Theorem 3.5 to the process $f_{2}^{\alpha}\left(\xi_{t}\right)$ for some $\alpha<1$.

First, we need the following important fact

Lemma 6.1 We have

$$
\mathbf{E}^{v}\left(f_{2}\left(\xi_{t+1}\right)-f_{2}\left(\xi_{t}\right) \mid \xi_{t}=S\right)=0
$$

for any $S \in \mathcal{D}$.

Proof. If $S \in \mathcal{D}_{0}$, then (6.3) is trivial. For $S \notin \mathcal{D}_{0}$ a direct computation gives

$$
\begin{aligned}
& f_{2}\left(S_{k}^{+r}\right)-f_{2}(S)=\frac{1}{2}\left(R_{k}+T_{k+1}+R_{k}^{2}-T_{k+1}^{2}\right)-\sum_{i=k+1}^{N} m_{i} R_{i}+\sum_{i=1}^{k} n_{i} T_{i} \\
& f_{2}\left(S_{k}^{-r}\right)-f_{2}(S)=\frac{1}{2}\left(R_{k}+T_{k+1}-R_{k}^{2}+T_{k+1}^{2}\right)+\sum_{i=k+1}^{N} m_{i} R_{i}-\sum_{i=1}^{k} n_{i} T_{i}
\end{aligned}
$$

for $k=0, \ldots, N$, and

$$
\begin{aligned}
& f_{2}\left(S_{k}^{+l}\right)-f_{2}(S)=\frac{1}{2}\left(-R_{k}-T_{k}+R_{k}^{2}-T_{k}^{2}\right)-\sum_{i=k}^{N} m_{i} R_{i}+\sum_{i=1}^{k} n_{i} T_{i} \\
& f_{2}\left(S_{k}^{-l}\right)-f_{2}(S)=\frac{1}{2}\left(-R_{k}-T_{k}-R_{k}^{2}+T_{k}^{2}\right)+\sum_{i=k}^{N} m_{i} R_{i}-\sum_{i=1}^{k} n_{i} T_{i}
\end{aligned}
$$


for $k=1, \ldots, N$.

Taking summation in (6.4)-(6.7) one gets 0 , thus finishing the proof of Lemma 6.1.

Then, from (6.5) we note that

$$
\left|f_{2}\left(S_{0}^{-r}\right)-f_{2}(S)\right| \geq \frac{T_{1}^{2}}{2}
$$

and from $(6.4)$

$$
\left|f_{2}\left(S_{N}^{+r}\right)-f_{2}(S)\right| \geq \frac{R_{N}^{2}}{2}
$$

These two inequalities give us that there exist a constant $C>0$ such that

$$
\mathbf{E}^{v}\left(\left(f_{2}\left(\xi_{t+1}\right)-f_{2}\left(\xi_{t}\right)\right)^{2} \mid \xi_{t}=S\right) \geq \frac{C|S|^{4}}{N}
$$

for all $S$. Now, a very important observation is that the VM does not increase the number of blocks $N_{t}=N\left(\xi_{t}\right)$. So we have for all $S$

$$
\mathbf{E}^{v}\left(\left(f_{2}\left(\xi_{t+1}\right)-f_{2}\left(\xi_{t}\right)\right)^{2} \mid \xi_{t}=S\right) \geq C_{0}|S|^{4}
$$

with $C_{0}=C_{0}\left(S_{0}\right)=C / N\left(S_{0}\right)$.

Elementary calculus gives us that for $0<\alpha<1$ and for $|x| \leq 1$ there exists a positive constant $C_{1}$ such that

$$
(x+1)^{\alpha}-1 \leq \alpha x-C_{1} x^{2} .
$$

Using now considerations analogous to (5.12) and applying (6.12), Lemma 6.1 and (6.11) we get

$$
\mathbf{E}^{v}\left(\left(f_{2}\left(\xi_{t+1}\right)\right)^{\alpha}-\left(f_{2}\left(\xi_{t}\right)\right)^{\alpha} \mid \xi_{t}=S\right) \leq-C_{0} C_{1}\left(f_{2}(S)\right)^{\alpha-2}|S|^{4} .
$$

Applying Lemma 4.1, part ii), to the last inequality we get

$$
\mathbf{E}^{v}\left(\left(f_{2}\left(\xi_{t+1}\right)\right)^{\alpha}-\left(f_{2}\left(\xi_{t}\right)\right)^{\alpha} \mid \xi_{t}=S\right) \leq-16 C_{0} C_{1}\left(f_{2}(S)\right)^{\alpha-2 / 3}
$$

We apply Theorem 3.5 to the process $X_{t}=\left(f_{2}\left(\xi_{t}\right)\right)^{1 / 3}$ taking $\alpha$ to be close to 1 to finish the proof of (6.1).

Let us turn now to the proof of (6.2). We let the process start from configuration $S_{0}$ such that $N\left(S_{0}\right)=1$. Since this configuration is reachable 
from any other configuration, it is sufficient to prove (6.2) for this $S_{0}$. As it was mentioned before, the voter model does not make the number of blocks $N$ increase, so the process can be represented as $\xi_{t}=\left(n_{t}, m_{t}\right)$, which clearly is a random walk in $\mathbb{Z}_{+}^{2}$, and we are interested in the moment of hitting the boundary. Note that the transition probabilities of this random walk can be described like this: from the state $(n, m)$ the transition can occur to the states $(n+1, m),(n-1, m),(n, m+1),(n, m-1),(n+1, m-1)$ and $(n-1, m+1)$ with probabilities $1 / 6$.

Denote by $\tau_{n, m}$ the moment of hitting $\mathcal{D}_{0}$ (i.e. the boundary) provided that the starting point was $(n, m)$. To proceed, we need the following

Lemma 6.2 There exist two positive constants $\delta, C$, such that for any $n, m$

$$
\mathbf{P}\left\{\tau_{n, m}>\delta n^{2}\right\} \geq \frac{C m}{m+n}
$$

and

$$
\mathbf{P}\left\{\tau_{n, m}>\delta m^{2}\right\} \geq \frac{C n}{m+n} .
$$

Remark 6.1 It can be shown that Lemma 6.9 holds for any homogeneous random walk in $\mathbb{Z}_{+}^{2}$ with bounded jumps and zero drift in the interior of $\mathbb{Z}_{+}^{2}$.

Proof. Without loss of generality we can suppose that $n \leq m$. Then, to prove (6.14), we will prove a stronger fact:

$$
\mathbf{P}\left\{\tau_{n, m}>\delta n^{2}\right\} \geq C_{0}
$$

for some $C_{0}$. In fact, it is a classical result that a homogeneous random walk in $\mathbb{Z}_{+}^{2}$ with bounded jumps and zero drift in the interior with some uniformly positive probability cannot deviate by the distance $n$ from its initial position during the time $n^{2}$. To show how it can be proved formally, we denote by $\rho\left(\left(n_{1}, m_{1}\right),\left(n_{2}, m_{2}\right)\right)$ the Euclidean distance between the points $\left(n_{1}, m_{1}\right)$ and $\left(n_{2}, m_{2}\right)$. Let the process start from $(n, m)$, and denote $Y_{t}=\rho\left(\xi_{t},(n, m)\right)$. Then, it is straightforward to get that the process $Y_{t}$ satisfies the hypothesis of Lemma 2 from Aspandiiarov et al. (1996), so applying it, we finish the proof of (6.14). 
To prove (6.15), we need some additional notations. Denote

$$
\begin{aligned}
W^{i}(m) & =\left\{\left(n^{\prime}, m^{\prime}\right): \rho\left(\left(n^{\prime}, m^{\prime}\right),(m, m)\right) \leq m / 2+\sqrt{2}\right\}, \\
V^{i}(m) & =\left\{\left(n^{\prime}, m^{\prime}\right): m / 2<\rho\left(\left(n^{\prime}, m^{\prime}\right),(m, m)\right) \leq m / 2+\sqrt{2}\right\}, \\
W^{e}(m) & =\left\{\left(n^{\prime}, m^{\prime}\right): m / 2+\sqrt{2}<\rho\left(\left(n^{\prime}, m^{\prime}\right),(m, m)\right) \leq m\right\}, \\
V^{e}(m) & =\left\{\left(n^{\prime}, m^{\prime}\right): m-\sqrt{2}<\rho\left(\left(n^{\prime}, m^{\prime}\right),(m, m)\right) \leq m\right\} .
\end{aligned}
$$

Clearly, the set $V^{i}(m)$ is the boundary of $W^{i}(m)$, and the set $V^{e}(m)$ is the external boundary of $W^{e}(m)$.

We consider the two possible cases:

a) $(n, m) \in W^{i}(m)$,

b) $(n, m) \in W^{e}(m)$.

Case a): first, we denote $Y_{t}=\rho\left(\xi_{t},(m, m)\right)$. Then, we apply Lemma 2 from Aspandiiarov et al. (1996) to get that $\mathbf{P}\left\{\tau_{n, m}>\delta n^{2}\right\} \geq C_{1}$ for some $C_{1}$, and thus (6.15).

Case b): we keep the notation $Y_{t}$ from the previous paragraph. Denote by $p_{n, m}$ the probability of hitting the set $V^{i}(m)$ before the set $V^{e}(m)$, provided that the starting point is $(n, m)$. Our goal is to estimate this probability from below.

For $C>0$ consider the process $Z_{t}^{C}, t=0,1,2, \ldots$, defined in the following way:

$$
Z_{t}^{C}=\exp \left\{C\left(1-\frac{Y_{t}}{m}\right)\right\}=\exp \left\{C\left(1-\frac{\rho\left(\xi_{t},(m, m)\right)}{m}\right)\right\}
$$

$Z_{0}^{C}=\exp \{C n / m\}$. One can prove the following technical fact: there exists a constant $C$ (not depending on $m$ ) such that

$$
\mathbf{E}\left(Z_{t+1}^{C}-Z_{t}^{C} \mid \xi_{t}=\left(n^{\prime}, m^{\prime}\right)\right) \geq 0
$$

for any point $\left(n^{\prime}, m^{\prime}\right) \in W^{e}(m)$ and if $m$ is large enough. Indeed, using the fact that there exist two positive constants $C_{1,2}$ such that

$$
e^{-x}-1 \geq-x+C_{1} x^{2}
$$

on $|x|<C_{2}$, we write

$$
\begin{aligned}
& \mathbf{E}\left(Z_{t+1}^{C}-Z_{t}^{C} \mid \xi_{t}=\left(n^{\prime}, m^{\prime}\right)\right) \\
& \quad=\exp \left\{C\left(1-\frac{\left|n^{\prime}-m\right|}{m}\right)\right\} \mathbf{E}\left(\exp \left\{-\frac{C}{m}\left(Y_{t+1}-Y_{t}\right)\right\}-1 \mid \xi_{t}=\left(n^{\prime}, m^{\prime}\right)\right) \\
& \geq \frac{C}{m} \exp \left\{C\left(1-\frac{\left|n^{\prime}-m\right|}{m}\right)\right\} \mathbf{E}\left(-\left(Y_{t+1}-Y_{t}\right)+\frac{C_{1} C}{m}\left(Y_{t+1}-Y_{t}\right)^{2} \mid \xi_{t}=\left(n^{\prime}, m^{\prime}\right)\right) .
\end{aligned}
$$


Then, using properties of the process $Y_{t}$, one can complete the proof of (6.17).

Now, to estimate $p_{n, m}$, we make the sets $V^{i}(m)$ and $V^{e}(m)$ absorbing. Using that our random walk cannot overpass these sets, the process $Z_{t}^{C}$ converge as $t \rightarrow \infty$ to $Z_{\infty}^{C}$, so

$$
\begin{aligned}
\mathbf{E} Z_{\infty}^{C} & \geq p_{n, m} \exp \left\{\frac{C}{2}\right\}+\left(1-p_{n, m}\right) \\
& \geq \mathbf{E} Z_{0}^{C}=\exp \left\{\frac{C n}{m}\right\}
\end{aligned}
$$

and thus

$$
\begin{aligned}
p_{n, m} & \geq \frac{\exp \{C n / m\}-1}{\exp \{C / 2\}-1} \\
& \geq \frac{C}{\exp \{C / 2\}-1} \cdot \frac{n}{m} \geq \frac{2 C}{\exp \{C / 2\}-1} \cdot \frac{n}{m+n} .
\end{aligned}
$$

So, starting from the point $(n, m)$, with probability at least (6.18) the random walk hits the set $V^{i}(m)$. Then, from the case a) it follows that with uniformly positive probability it will take at least $\delta m$ steps to reach the external boundary $V^{e}(m)$, so we complete the proof of (6.15) and thus, of Lemma 6.2.

Now, supposing that (6.2) does not hold, we have (denoting $\tau:=\tau\left(S_{0}\right)$ and $a \wedge b:=\min \{a, b\})$

$$
\begin{aligned}
\mathbf{E} \tau^{3 / 2+\varepsilon} \geq & \mathbf{E}\left(\tau^{3 / 2+\varepsilon} \mathbf{1}_{\{\tau \geq t\}}\right)=\mathbf{E}\left(\left(t+\tau_{\xi_{t}}\right)^{3 / 2+\varepsilon} \mathbf{1}_{\left\{\xi_{s} \notin \mathcal{D}_{0} \text { for all } s \leq t\right\}}\right) \\
\geq & \frac{1}{2} \mathbf{E}\left(\left(t+\delta n_{t}^{2}\right)^{3 / 2+\varepsilon} \frac{C m_{t}}{m_{t}+n_{t}} \mathbf{1}_{\left\{\xi_{s} \notin \mathcal{D}_{0} \text { for all } s \leq t\right\}}\right) \\
& \quad+\frac{1}{2} \mathbf{E}\left(\left(t+\delta m_{t}^{2}\right)^{3 / 2+\varepsilon} \frac{C n_{t}}{m_{t}+n_{t}} \mathbf{1}_{\left\{\xi_{s} \notin \mathcal{D}_{0} \text { for all } s \leq t\right\}}\right) \\
\geq & \delta^{\prime} C^{\prime} \mathbf{E}\left(\left(n_{t}^{2+\varepsilon^{\prime}} m_{t}+m_{t}^{2+\varepsilon^{\prime}} n_{t}\right) \mathbf{1}_{\left\{\xi_{s} \notin \mathcal{D}_{0} \text { for all } s \leq t\right\}}\right) \\
= & \delta^{\prime} C^{\prime} \mathbf{E}\left(\left(n_{t \wedge \tau}^{2+\varepsilon^{\prime}} m_{t \wedge \tau}+m_{t \wedge \tau}^{2+\varepsilon^{\prime}} n_{t \wedge \tau}\right)\right) \\
= & C^{\prime \prime} \mathbf{E}\left(f_{2}\left(\xi_{t \wedge \tau}\right)\right)^{1+\varepsilon^{\prime \prime}} .
\end{aligned}
$$

for some constants $\delta^{\prime}, \varepsilon^{\prime}, C^{\prime}, \varepsilon^{\prime \prime}$ and $C^{\prime \prime}$.

From (6.19) we get that the family $\left\{f_{2}\left(\xi_{t \wedge \tau}\right)\right\}$ is uniformly integrable as $t \rightarrow \infty$, so $\mathbf{E} f_{2}\left(\xi_{t}\right) \rightarrow \mathbf{E} f_{2}\left(\xi_{\tau}\right)=0$. But this obviously contradicts to Lemma 6.1. 


\section{$7 \quad$ Hybrid process}

As it was proved before, the $\operatorname{EP}(p)$ is transient when $p \leq 1 / 2$, and $\mathrm{VM}$ is ergodic. Now, what will happen if we combine them? The following theorems give a (not complete) answer to this question.

Theorem 7.1 There exists $\beta_{0}<1$ such that for any $p$ the process $\operatorname{HP}(\beta, p)$ is ergodic for all $\beta>\beta_{0}$.

Theorem 7.2 For any $\beta>0$ and $p \geq 1 / 2$ the process $H P(\beta, 1 / 2)$ is ergodic.

We also formulate the following plausible conjecture. Its not completely rigorous proof will be presented in Section 7.3.

Conjecture 7.1 For any $p<1 / 2$ there exists $\beta_{0}=\beta_{0}(p)>0$ such that the process $H P(\beta, p)$ is not ergodic for $\beta<\beta_{0}$.

\subsection{Proof of Theorem 7.1}

We use the notations introduced in Section 1 . Direct computations yield

$$
\begin{aligned}
& f_{1}\left(S_{k}^{+l}\right)-f_{1}(S)=R_{k}-T_{k}-1, \\
& f_{1}\left(S_{k}^{-l}\right)-f_{1}(S)=-R_{k}+T_{k}-1, \\
& f_{1}\left(S_{k}^{+r}\right)-f_{1}(S)=R_{k}-T_{k+1}, \\
& f_{1}\left(S_{k}^{+r}\right)-f_{1}(S)=-R_{k}+T_{k+1},
\end{aligned}
$$

SO

$$
\mathbf{E}^{v}\left(f_{1}\left(\xi_{t+1}\right)-f_{1}\left(\xi_{t}\right) \mid \xi_{t}=S\right)=-\frac{N}{2 N+1} .
$$

Combining this with (5.7), we get that there exists a positive number $C=C(\beta)$ such that

$$
\begin{aligned}
\mathbf{E}_{\beta, p}^{h}\left(f_{1}\left(\xi_{t+1}\right)-f_{1}\left(\xi_{t}\right) \mid \xi_{t}=S\right) & =-\frac{1}{2 N+1}(\beta N-(1-\beta)((q-p) N+q)) \\
& <-C(\beta)
\end{aligned}
$$

for $\beta>2 / 3$. Applying Theorem 3.1, we finish the proof. 


\subsection{Proof of Theorem 7.2}

To prove the desired result, we are going to apply Theorem 3.1 to the function $\varphi(S):=\left(f_{2}(S)\right)^{\alpha}$ for some $\alpha<1$.

First, we prove the theorem for the case $p=1 / 2$.

Inserting $p=q=1 / 2$ into (5.3), we obtain for the step of $\operatorname{EP}(1 / 2)$

$$
\mathbf{E}_{1 / 2}^{e}\left(f_{2}\left(\xi_{t+1}\right)-f_{2}\left(\xi_{t}\right) \mid \xi_{t}=S\right)=\frac{1}{2}
$$

It is elementary to get that for $\alpha \in(0,1)$

$$
(x+1)^{\alpha}-1 \leq \alpha x
$$

for all $x \geq-1$. Using (7.3), (7.4) and (6.12), we get

$$
\mathbf{E}_{1 / 2}^{e}\left(\left(f_{2}\left(\xi_{t+1}\right)\right)^{\alpha}-\left(f_{2}\left(\xi_{t}\right)\right)^{\alpha} \mid \xi_{t}=S\right) \leq \frac{\alpha\left(f_{2}(S)\right)^{\alpha-1}}{2} .
$$

Now, let us make the necessary estimate for the step of VM. Here we will need a bound which is more accurate than (6.10):

Lemma 7.1 There exists $C^{\prime}>0$ such that

$$
\mathbf{E}^{v}\left(\left(f_{2}\left(\xi_{t+1}-f_{2}\left(\xi_{t}\right)\right)^{2} \mid \xi_{t}=S\right) \geq C^{\prime}|S|^{16 / 5}\right.
$$

Proof. To calculate exactly the left-hand side of (7.6), one has to square (6.4)-(6.7), sum them up and divide by $4 N+2$. But this calculation appears to be too difficult; so we will only obtain a lower bound. Denote $\Delta_{k}=$ $f_{2}\left(S_{k}^{+r}\right)-f_{2}(S)$, so

$$
\mathbf{E}^{v}\left(\left(f_{2}\left(\xi_{t+1}-f_{2}\left(\xi_{t}\right)\right)^{2} \mid \xi_{t}=S\right) \geq \frac{1}{4 N+2} \sum_{i=1}^{N} \Delta_{i}^{2} .\right.
$$

By simple algebraic calculations, one gets from (6.4) that

$$
\Delta_{i+1}-\Delta_{i} \geq N
$$

for $i=0, \ldots, N-1$. From (6.4) one gets also that $\Delta_{0}<0$ and $\Delta_{N}>0$, so denote $L=\min \left\{k: \Delta_{k-1}<0, \Delta_{k} \geq 0\right\}$. Using (7.8), we get

$$
\begin{aligned}
\sum_{i=1}^{N} \Delta_{i}^{2} & \geq \sum_{i=0}^{L-1}(N(L-i-1))^{2}+\sum_{i=L}^{N}(N(i-L))^{2} \\
& \geq N^{2} \sum_{i=1}^{N / 2} i^{2} \geq C_{1} N^{5}
\end{aligned}
$$


for some $C_{1}$, so by $(7.7)$ we get that

$$
\mathbf{E}^{v}\left(\left(f_{2}\left(\xi_{t+1}-f_{2}\left(\xi_{t}\right)\right)^{2} \mid \xi_{t}=S\right) \geq C_{2} N^{4}\right.
$$

for some $C_{2}$. Combining this with (6.10), we get

$$
\mathbf{E}^{v}\left(\left(f_{2}\left(\xi_{t+1}-f_{2}\left(\xi_{t}\right)\right)^{2} \mid \xi_{t}=S\right) \geq \max \left\{C_{2} N^{4}, \frac{C|S|^{4}}{N}\right\} \geq C^{4 / 5} C_{2}^{1 / 5}|S|^{16 / 5},\right.
$$

thus proving Lemma 7.1 .

Remark 7.1 The exponent 16/5 in Lemma 7.1 is the best possible; to see this, one may take a configuration $S$ with $n_{1}=m_{N}=N^{5 / 4}$ and $n_{2}=\cdots=$ $n_{N}=m_{1}=\cdots=m_{N-1}=1$ and compute the left-hand side of (7.6).

We continue proving Theorem 7.2. Analogously to (5.12), using (6.12) together with Lemmas 6.1 and 7.1, we get for some positive constant $C_{2}$

$$
\mathbf{E}^{v}\left(\left(f_{2}\left(\xi_{t+1}\right)\right)^{\alpha}-\left(f_{2}\left(\xi_{t}\right)\right)^{\alpha} \mid \xi_{t}=S\right) \leq-C_{2}\left(f_{2}(S)\right)^{\alpha-2}|S|^{16 / 5}
$$

So, for $\operatorname{HP}(\beta, 1 / 2)$, combining (7.5) with $(7.9)$ and using that $|S|^{16 / 5} \geq$ $2^{16 / 5}\left(f_{2}(S)\right)^{16 / 15}$ because of part ii) of Lemma 4.1, we get for $14 / 15<\alpha<1$

$$
\begin{aligned}
& \mathbf{E}_{\beta, 1 / 2}^{h}\left(\varphi\left(\xi_{t+1}\right)-\varphi\left(\xi_{t}\right) \mid \xi_{t}=S\right) \\
& \quad=\mathbf{E}_{\beta, 1 / 2}^{h}\left(\left(f_{2}\left(\xi_{t+1}\right)\right)^{\alpha}-\left(f_{2}\left(\xi_{t}\right)\right)^{\alpha} \mid \xi_{t}=S\right) \\
& \quad \leq(1-\beta) \frac{\alpha\left(f_{2}(S)\right)^{\alpha-1}}{2}-\beta C_{2}\left(f_{2}(S)\right)^{\alpha-2}|S|^{16 / 5} \\
& \quad \leq(1-\beta) \frac{\alpha\left(f_{2}(S)\right)^{\alpha-1}}{2}-2^{16 / 5} \beta C_{2}\left(f_{2}(S)\right)^{\alpha-14 / 15} \\
& =-\left(f_{2}(S)\right)^{\alpha-14 / 15}\left[2^{16 / 5} \beta C_{2}-\frac{(1-\beta) \alpha}{2}\left(f_{2}(S)\right)^{-1 / 15}\right]<-1
\end{aligned}
$$

for all but a finite number of $S$ 's (indeed, the expression in the square brackets is of order of positive constant for all but finitely many $S$, and the fact that $\alpha>14 / 15$ guarantees that the absolute value of the left-hand side of $(7.10)$ is large enough for all but a finite number of $S$ 's). Applying Theorem 3.1, we finish the proof of Theorem 7.2 for $p=1 / 2$.

Now, when $p>1 / 2$, using (5.4) and Lemma 6.1 we get that for any $\varepsilon>0$ $\mathbf{E}_{\beta, p}^{h}\left(f_{2}\left(\xi_{t+1}\right)-f_{2}\left(\xi_{t}\right) \mid \xi_{t}=S\right)=(1-\beta) \mathbf{E}_{p}^{e}\left(f_{2}\left(\xi_{t+1}\right)-f_{2}\left(\xi_{t}\right) \mid \xi_{t}=S\right)<-(1-\beta) \varepsilon$ for all but finite number of $S$, and we apply Theorem 3.1 again. 
Remark 7.2 Using the technique of Section 6, it is possible to get that there exists some $p_{0}=p_{0}(\beta)>1$ such that for the process $\operatorname{HP}(\beta, 1 / 2)$ we have that $\mathbf{E}\left(\tau\left(S_{0}\right)\right)^{p}<\infty$ for all $p<p_{0}$. By using the technique of Menshikov and Popov (1995), one can get polynomial bounds on the decay of the stationary measure.

\subsection{Nonergodicity}

Here we will present an argument in the favor of the validity of Conjecture 7.1 .

We rewrite (7.2) as

$$
\begin{aligned}
\mathbf{E}_{\beta, p}^{h}\left(f_{1}\left(\xi_{t+1}\right)-f_{1}\left(\xi_{t}\right) \mid \xi_{t}=S\right) & =\frac{1}{2 N+1}(-\beta N+(1-\beta)((q-p) N+q)) \\
& \geq \frac{N}{2 N+1}((1-\beta)(q-p)-\beta)>0 \quad(7.11)
\end{aligned}
$$

when $\beta<\frac{q-p}{2 q}$. Unfortunately, because the VM does not have the property (3.5), we cannot apply Theorem 3.4. Moreover, it is still very unclear to us, if the process is transient in this case. So instead we shall explain why we believe it is not ergodic.

We need the following three lemmas:

Lemma 7.2 Let $\xi_{t}, t=0,1,2, \ldots$ be a Markov chain on a countable state space $X$, let $0 \in X$ be an absorbing state, and define $\tau:=\min \left\{t: \xi_{t}=0\right\}$ to be the hitting time of 0 . Suppose that for any starting point $x$ we have $f(x):=\mathbf{E}_{x} \tau<\infty$. Then

$$
\mathbf{E} f\left(\xi_{t}\right) \rightarrow 0
$$

as $t \rightarrow \infty$.

Proof. Let $x_{0}$ be the starting position of the Markov chain. It is straightforward to get

$$
\mathbf{E}\left(f\left(\xi_{t+1}\right)-f\left(\xi_{t}\right) \mid \xi_{t}=x\right)=-\mathbf{1}_{\{x \neq 0\}},
$$

so, taking expectation in (7.13), we get

$$
\mathbf{E}_{x_{0}} f\left(\xi_{t+1}\right)-\mathbf{E}_{x_{0}} f\left(\xi_{t}\right)=-\mathbf{P}_{x_{0}}\{\tau>t\} .
$$

Taking summation in (7.14), we obtain

$$
\mathbf{E}_{x_{0}} f\left(\xi_{t+1}\right)=f\left(x_{0}\right)-\sum_{i=0}^{t} \mathbf{P}_{x_{0}}\{\tau>i\} \rightarrow 0
$$


as $t \rightarrow \infty$, thus completing the proof of Lemma 7.2 .

Lemma 7.3 Let $\xi_{t}, t=0,1,2, \ldots$ be a Markov chain on a countable state space $X$, and let $0 \in X$ be an absorbing state. Let $x_{0}$ be the starting position of the Markov chain, $\tau$ be the moment of hitting 0 , and suppose that $\mathbf{E}_{x_{0}} \tau<$ $\infty$ for all $x_{0}$. Let $f(x)$ be some nonnegative function on $X$ such that for some constant $K$

$$
\mathbf{E}\left(f\left(\xi_{t+1}\right)-f\left(\xi_{t}\right) \mid \xi_{t}=x\right) \leq K
$$

for all $x \neq 0$. Then there exists a constant $M$ such that $\mathbf{E} f\left(\xi_{t}\right)<M$ for all $t$.

Proof. The proof is analogous to that of Lemma 7.2: first, we rewrite (7.15) as

$$
\mathbf{E}\left(f\left(\xi_{t+1}\right)-f\left(\xi_{t}\right) \mid \xi_{t}=x\right) \leq K \mathbf{1}_{\{\tau>t\}}
$$

for all $x$. So,

$$
\mathbf{E}_{x_{0}} f\left(\xi_{t+1}\right)-\mathbf{E}_{x_{0}} f\left(\xi_{t}\right) \leq K \mathbf{P}_{x_{0}}\{\tau>t\}
$$

and, taking summation in (7.17), we get

$$
\mathbf{E}_{x_{0}} f\left(\xi_{t+1}\right) \leq f\left(x_{0}\right)+K \sum_{i=0}^{t} \mathbf{P}_{x_{0}}\{\tau>i\} \leq f\left(x_{0}\right)+K \mathbf{E}_{x_{0}} \tau .
$$

Denoting $M:=f\left(x_{0}\right)+K \mathbf{E}_{x_{0}} \tau$, we finish the proof of Lemma 7.3.

Analogously to Lemmas 7.2 and 7.3 , we can prove the following lemma (which is, in fact, an adaptation of Lemma 2.2 from Menshikov and Popov (1995) to our situation)

Lemma 7.4 Let $\xi_{t}, t=0,1,2, \ldots$ be a Markov chain on a countable state space $X$, let $0 \in X$ be an absorbing state, $x_{0}$ be the starting point, and $\tau$ be the moment of hitting 0 , and suppose that $\mathbf{E}_{x_{0}} \tau<\infty$ for all $x_{0}$. Let $f(x)$ be some nonnegative function on $X$ such that $\mathbf{E}_{x_{0}} f\left(\xi_{t}\right) \rightarrow 0$ as $t \rightarrow \infty$, and for some positive constant $K$

$$
\mathbf{E}\left(f\left(\xi_{t+1}\right)-f\left(\xi_{t}\right) \mid \xi_{t}=x\right) \geq-K
$$

for all $x$. Then $\mathbf{E}_{x_{0}} \tau \geq f\left(x_{0}\right) / K$. 
So, let us take a hybrid process $\operatorname{HP}(\beta, p)$ which satisfies (7.11). We suppose that it is ergodic and try to get a contradiction. Define $\varphi_{\beta, p}(S)$ to be the mean hitting time of $\mathcal{D}_{0}$ starting from $S$, i.e. $\varphi_{\beta, p}(S)=\mathbf{E}_{\beta, p}^{h} \tau(S)$. We will prove the following

Lemma 7.5 For any $p>1 / 2, \beta$, there exists a positive constant $C=C(\beta, p)$ such that

$$
\varphi_{\beta, p}(S) \geq C f_{1}(S)
$$

Proof. From (5.3) and Lemma 6.1 we get that for $p>1 / 2$ and any $\beta$

$$
\mathbf{E}_{\beta, p}^{h}\left(f_{2}\left(\xi_{t+1}\right)-f_{2}\left(\xi_{t}\right) \mid \xi_{t}=S\right) \leq \frac{1}{2}
$$

Applying Lemma 7.3, we get that there exists a constant $M$ such that $\mathbf{E}_{\beta, p}^{h} f_{2}\left(\xi_{t}\right)<M$ for all $t$. Using Lemma 4.1 iii), we see that $\mathbf{E}_{\beta, p}^{h} f_{1}\left(\xi_{t}\right) \rightarrow 0$ as $t \rightarrow \infty$. Applying Lemma 7.4, we complete the proof of Lemma 7.5.

Conjecture 7.2 Lemma 7.5 holds for any $p$.

We failed to prove the above conjecture. Intuitively, $\varphi_{\beta, p}(S)$ grows when $p$ decrease and the monotonicity argument might be applicable to prove this fact. For the pure exclusion process, this argument follows from the basic coupling (see Liggett (1985, Section VIII.2)). When the voter model is added, this coupling does not work.

Now, if we suppose this to be true, the rest of the proof is straightforward. If the process $\operatorname{HP}(\beta, p)$ is ergodic, then the function $\varphi_{\beta, p}(S)$ is well defined, so, by Lemma 7.2, $\mathbf{E}_{\beta, p}^{h} \varphi_{\beta, p}\left(\xi_{t}\right) \rightarrow 0$ as $t \rightarrow \infty$. Thus, using (7.19), we get that $\mathbf{E}_{\beta, p}^{h} f_{1}\left(\xi_{t}\right) \rightarrow 0$. But this obviously contradicts to (7.11).

\section{Continuous time}

In this section we show how Theorem 1.1 follows from the theorems proved in the last three sections.

Observe first that the transience is a property of the skeleton of a Markov process. In our case the skeleton is the process $\xi_{n}=\eta_{\tau_{n}}$, as defined in Section 2. Notice that according to this definition, $\xi_{n+1}$ may be the same as $\xi_{n}$; this deviates a bit from the usual notion of skeleton. In our version of skeleton the exit time of a configuration is a geometric random variable 
with parameter bigger than $\beta+(1-\beta) \min \{p, q\}$. This implies that the skeleton can not get stacked. Hence Theorem 5.2 which states the transience for the discrete-time exclusion process with $p \leq 1 / 2$ implies the same for the continuous-time process. This shows the transient part of item 1 of Theorem 1.1.

To prove that the ergodicity for the discrete-time process implies the ergodicity for the continuous-time one, let $\eta \in \mathcal{D}_{0}$, let $S$ be the class of equivalence of $\eta$ and write

$$
\tau_{c}(\eta)=\sum_{n=1}^{\tau(S)}\left(\tau_{n}-\tau_{n-1}\right)
$$

where we recall that $\tau_{c}(\eta)$ and $\tau(S)$ are the hitting times of $\mathcal{D}_{0}$ for the continuous and discrete time processes starting from $\eta$ and $S$ respectively and $\tau_{n}$ is the instant of the $n$-th attempted jump of the continuous process $\eta_{t}$, as defined in (2.1). Given the past up to $\tau_{n}, \tau_{n+1}-\tau_{n}$ is an exponential random variable with rate bigger than 1 - the worst case, the configurations belonging to $\mathcal{D}_{0}$. Hence, $\tau_{n+1}-\tau_{n}$ is stochastically bounded below by an exponential random variable of rate 1 independent of everything. This implies that

$$
\mathbf{E} \tau_{c}(\eta) \leq \mathbf{E} \tau(\eta)
$$

Since the ergodicity is equivalent to the finiteness of the expected return time for any given configuration then the ergodicity for the discrete-time process implies the same for the continuous-time one. With this argument Theorem 5.1 implies the ergodic part of item 1 of Theorem 1.1 and Theorems 7.1 and 7.2 imply item 2 of Theorem 1.1.

The argument above implies a stronger statement for the pure voter model. Let $\beta=0$ and observe that for any $\eta \notin \mathcal{D}_{0}$, there are at least three discrepancies. Hence, for the voter model,

$$
\tau_{c}(\eta) \leq \sum_{n=1}^{\tau(S)}\left(\tau_{n}^{\prime}-\tau_{n-1}^{\prime}\right) .
$$

where $\left(\tau_{n}^{\prime}-\tau_{n-1}^{\prime}\right)$ are independent exponentially distributed with parameter 3 and independent of $\tau(S)$. This together with Lemma 8.1 below show that the first part of (1.3) follows from (6.1).

We now show how to obtain the second part of (1.3) from (6.2). Let $\mathcal{D}_{1}$ be the set of configurations on $\mathcal{D}$ having exactly three discrepancies (that is, 10 , 
01 and 10). This means that in the representation (4.1) the configurations belonging to $\mathcal{D}_{1}$ have $N=1$, that is only one finite 0 -block and one finite 1-block. The transition rate for configurations in $\mathcal{D}_{1}$ in the voter model is exactly 3 . If the process is in $\mathcal{D}_{1}$ then it can only either stay in $\mathcal{D}_{1}$ or jump to $\mathcal{D}_{0}$. Hence, for $\eta \in \mathcal{D}_{1}$,

$$
\tau_{c}(\eta)=\sum_{n=1}^{\tau(S)}\left(\tau_{n}^{\prime}-\tau_{n-1}^{\prime}\right)
$$

Lemma 8.1 below shows that (6.2) implies that the left hand side of (8.4) is infinite for any $\eta \in \mathcal{D}_{1}$. As argued before, any configuration in $\mathcal{D}_{1}$ is

reachable from any other, hence the same is valid for any $\eta \in \mathcal{D} \backslash \mathcal{D}_{0}$. This shows the second part of (1.3).

Lemma 8.1 Let $\tau$ be a positive integer random variable and $\tau_{i}$ be nonnegative independent random variables with the exponential distribution and independent of $\tau$. Then for any $p>0$

$$
\mathbf{E}\left(\sum_{n=1}^{\tau} \tau_{n}\right)^{p}<\infty \quad \text { if and only if } \mathbf{E} \tau^{p}<\infty .
$$

Proof. By independence,

$$
\mathbf{E}\left(\sum_{n=1}^{\tau} \tau_{n}\right)^{p}=\sum_{n} \mathbf{E}\left(\sum_{i=1}^{n} \tau_{i}\right)^{p} \mathbf{P}(\tau=n) .
$$

But

$$
\mathbf{E}\left(\sum_{i=1}^{n} \tau_{i}\right)^{p}=\frac{\Gamma(n+p)}{\Gamma(n)}
$$

which is of the order of $n^{p}$.

\section{Final remarks}

Let us give several remarks with respect to extensions of our results to a nonnearest-neighbor case. For a nonnearest-neighbor voter model, we failed to find an analogue of Lemma 6.1. Exactly to say, in this case, Lemma 6.1 is incorrect for $f_{2}$ as stated, and we could not find a substitute for $f_{2}$ that would 
provide a relevant information both for this voter model and for the hybrid process constructed via mixing this voter model with an exclusion process of any range. When the hybrid process consists of a nearest neighbor voter model and a finite range exclusion process, an analogue of the statement $2 \mathrm{i}$ ) of Theorem 1.1 may be obtained by an appropriate, although straightforward, modification of our arguments. Anything beyond this result was not possible. A reason for this was again, our failure in finding the substitutes of $f_{1}$ and $f_{2}$ that would work for this case as well as $f_{1}$ and $f_{2}$ have worked for the nearest neighbor system.

The presented above results show that, to a certain extent, the success of our methods depend on the correct choice of the Lyapunov function.

\section{Acknowledgements}

The authors thank FAPESP and CNPq for financial support.

\section{References}

[1] S. Aspandiiarov, R. Iasnogorodsky and M.V. Menshikov (1996) Passagetime moments for non-negative stochastic processes and an application to reflected random walks in a quadrant. Ann. Probab. 24 (2), 932-960.

[2] M. Bramson, P. Calderoni, A. De Masi, P.A. Ferrari, J. Lebowitz and R. Schonmann (1986) Microscopic selection principle for a diffusionreaction equation. J. Statist. Phys. 45 (5/6), 905-920.

[3] C. Cammarota and P.A. Ferrari (1991) Invariance principle for the branching exclusion process. Stochastic Process. Appl. 38 (1), 1-11.

[4] J.T. Cox and R. Durrett (1995) Hybrid zones and voter model interfaces. Bernoulli 1 (4), 343-370.

[5] A. De Masi, P.A. Ferrari and J. Lebowitz (1986) Reaction-diffusion equations for interacting particle systems. J. Statist. Phys. 44 (3/4), 589-644.

[6] B. Derrida, S. Goldstein, J. L. Lebowitz and E. Speer (1998) Shift equivalence of measures and the intrinsic structure of shocks in the asymmetric simple exclusion process. J. Statist. Phys. 93 (3/4), 547-571. 
[7] R. Durrett (1988) Lecture Notes on Particle Systems and Percolation. Belmont, CA: Wadsworth.

[8] R. Durrett (1995) Ten Lectures on Particle Systems. St. Flour Lecture Notes, Lecture Notes in Math. New York: Springer-Verlag.

[9] G. Fayolle, V.A. Malyshev and M.V. Menshikov (1995) Topics in the Constructive Theory of Countable Markov Chains. Cambridge University Press.

[10] P.A. Ferrari (1992) Shock fluctuations in asymmetric simple exclusion. Probab. Theory Related Fields 91 (1), 81-101.

[11] P.A. Ferrari (1994) Shocks in one-dimensional processes with drift. In: G. Grimmett (ed.), Probability and Phase Transition (Cambridge, 1993), NATO Adv. Sci. Ins. Ser. C, Math. Phys. Sci. 420, 35-48, Dordrecht. Kluwer Acad. Publ.

[12] P.A. Ferrari (1996) Growth processes on a strip. In: Disordered systems (Temuco, 1991/1992) 87-111. Travaux en Cours, 53. Hermann, Paris.

[13] P.A. Ferrari, C. Kipnis and S. Saada (1991) Microscopic structure of travelling waves in the asymmetric simple exclusion process. Ann Probab. 19 (1), 226-244.

[14] T.M. Liggett (1976) Coupling the simple exclusion process. Ann. Probab. 4, 339-356.

[15] T.M. Liggett (1985) Interacting Particle Systems. Springer, Berlin.

[16] T.M. Liggett (1999) Stochastic Interacting Systems. Exclusion, voter and contact processes. Springer, Berlin.

[17] F.P. Machado (1998) Asymptotic shape for the branching exclusion process. Markov Processes Relat. Fields 4 (4), 535-547.

[18] V.A. Malyshev (1998) Random grammars. (Russian) Uspekhi Mat. Nauk 53 (2(320)), 107-134; translation in Russian Math. Surveys 53 (2), 345370.

[19] M.V. Menshikov and S.Yu. Popov (1995) Exact power estimates for countable Markov chains. Markov Processes Relat. Fields 1 (1), 57-78. 